\title{
Towards a Rice Theorem on Traces of Cellular Automata $^{\star}$
}

\author{
Julien Cervelle and Pierre Guillon \\ Institut Gaspard Monge, Université de Marne la Vallée \\ 77454 Marne la Vallée Cedex 2, France \\ \{julien.cervelle,pierre.guillon\}@univ-mlv.fr
}

\begin{abstract}
The trace subshift of a cellular automaton is the subshift of all possible columns that may appear in a space-time diagram. We prove the undecidability of a rather large class of problems over trace subshifts of cellular automata.
\end{abstract}

Keywords: discrete-time dynamical systems, cellular automata, symbolic dynamics, formal languages, computability, decidability.

\section{Introduction}

Cellular automata are well-known formal models for complex systems. They are used in a huge variety of different scientific fields including mathematics, physics and computer science.

A cellular automaton (CA for short) consists in an infinite number of identical cells arranged on a regular lattice. All cells evolve synchronously according to their own state and those of their neighbors.

Despite the apparent simplicity of the definition of the system, one can observe very complex behaviors. Many attempts have been made to classify them: strictly in topological terms [1], emphasizing limit sets [2], considering computability [3], or yet studying the language appearing in cell evolution [4]. Most behaviors have been proved undecidable: concerning the attractors (nilpotency in [5], other properties in [6]), the equicontinuity classification [7], or universality [8].

Our approach concerns the languages that can be associated to a CA and especially the trace subshift which is the set of all possible sequences of the states a particular cell takes during the evolution. Motivations come both from classical symbolic dynamics but also from physics. Indeed, when observing natural phenomena due to physical constraints, one can keep trace only of a finite number of measurements. This set of measurements takes into account only a minor part of the parameters ruling the phenomenon under investigation. Hence,

\footnotetext{
* This work has been supported by the Interlink/MIUR project "Cellular Automata: Topological Properties, Chaos and Associated Formal Languages", by the ANR Blanc "Projet Sycomore".
} 
to some extent, what is observed is the "trace" of the phenomenon left on the instruments.

We try to prove a "Rice Theorem" for trace subshifts, similarly to what is done in $[6,9]$ for limits sets and tilings, that is to say the undecidability of non-trivial properties over the trace subshift given a CA.

The paper is organized as follows. Section 2 to 4 are devoted to definitions and main concepts of the paper. Section 5 study the case of a Rice theorem for a simpler definition of trace called "subtrace". Section 6 extends the result to the "trace" case.

\section{Definitions}

Let $\mathbb{N}^{*}=\mathbb{N} \backslash\{0\}$. For $i, j \in \mathbb{N}$ with $i \leq j,[i, j]$ denotes the set of integers between $i$ and $j$ inclusive. For any function $F$ from $A^{\mathbb{Z}}$ into itself, $F^{n}$ denotes the $n$-fold composition of $F$ with itself.

Languages. Let $A$ be a finite alphabet with at least two letters. A word is a finite sequence of letters $w=w_{0} \ldots w_{|w|-1} \in A^{*}$. A factor of a word $w=$ $w_{0} \ldots w_{|w|-1} \in A^{*}$ is a word $w_{[i, j]}=w_{i} \ldots w_{j}$, for $0 \leq i \leq j<|w|$. We note $w_{[i, j]} \sqsubset w$. A language on alphabet $A$ is a set of words on alphabet $A$. Given two languages $C, D \subset A^{*}, C D$ denotes their concatenation, When no confusion is possible, given a word $w$, we also note $w$ the language $\{w\}$.

Configurations. A configuration is a bi-infinite sequence of letters $x \in A^{\mathbb{Z}}$. The set $A^{\mathbb{Z}}$ of configurations is the phase space. The definition of factor can be naturally extended to configurations: for $x \in A^{\mathbb{Z}}$ and $i \leq j, x_{[i, j]}=x_{i} \ldots x_{j} \sqsubset x$. If $u \in A^{*}$, then $u^{\omega}$ is the infinite word of $A^{\mathbb{N}}$ consisting in periodic repetitions of $u$ and ${ }^{\omega} u^{\omega}$ is the configuration of $A^{\mathbb{Z}}$ consisting in periodic repetitions of $u$.

Topology. We endow the phase space with the Cantor topology. For $j, k \in \mathbb{N}$ and a finite set $W$ of words of length $j$, we note $[W]_{k}$ the set $\left\{x \in A^{\mathbb{Z}} \mid x_{[k, k+j-1]} \in W\right\}$. Such a set is called a cylinder. We note $[W]_{k}^{C}$ the complement of the cylinder $[W]_{k}$. Cylinders form a base of open sets for Cantor topology.

Cellular automata. A (one-dimensional two-sided) cellular automaton (CA for short) is a parallel synchronous computation model consisting in cells distributed over the regular lattice $\mathbb{Z}$. Each cell $i \in \mathbb{Z}$ of the configuration $x \in A^{\mathbb{Z}}$ has a state $x_{i}$ in the finite alphabet $A$, which evolves depending on the state of their neighbors: $\left.F(x)_{i}=f\left(x_{[i-m, i-m+d-1]}\right)\right)$, where $f: A^{d} \rightarrow A$ is the local rule, $m \in \mathbb{Z}$ the anchor and $d \in \mathbb{N}^{*}$ the diameter of the CA. By abuse of notation, we use the cellular automaton as its global function $F: A^{\mathbb{Z}} \rightarrow A^{\mathbb{Z}}$. The orbit of initial configuration $x \in A^{\mathbb{Z}}$ is the sequence of the configurations $\left(F^{j}(x)\right)_{j \in \mathbb{N}}$. Usually they are graphically represented by a two-dimensional space-time diagram, like in Figure 1.

The shift $\sigma: A^{\mathbb{Z}} \rightarrow A^{\mathbb{Z}}$ is a particular CA defined by $\sigma(x)_{i}=x_{i+1}$ for every $x \in A^{\mathbb{Z}}$ and $i \in \mathbb{Z}$ which shifts configurations to the left. If the alphabet contains 
a special letter 0 , the null $C A$ null $: A^{\mathbb{Z}} \rightarrow A^{\mathbb{Z}}$ is another particular CA defined by $\operatorname{null}(x)_{i}=0$. Its image set is $\left\{{ }^{\omega} 0^{\omega}\right\}$.

Any local rule $f$ of a $\mathrm{CA}$ can be extended naturally to an application on words $f(w)=\left(f\left(w_{[i, i+d-1]}\right)\right)_{0 \leq i \leq|w|-d}$, for all $w \in A^{*} A^{d}$.

Finally, if a CA has anchor 0 , it is called an one-way cellular automata (OCA for short), since the new state of a cell only depends on its right neighbors. Hence information can only flow from right to left.

Subshifts. The one-sided shift (or simply shift when no confusion is possible), also noted $\sigma$ by abuse of notation is the self-map of $A^{\mathbb{N}}$ such that $\sigma(z)_{i}=z_{i+1}$, for every $z \in A^{\mathbb{N}}$ and $i \in \mathbb{N}$. A one-sided subshift $\Sigma \subset A^{\mathbb{N}}$ is a $\sigma$-stable closed set of infinite words. The language of $\Sigma$ is $\mathcal{L}(\Sigma)=\left\{w \in A^{*} \mid \exists z \in \Sigma, w \sqsubset z\right\}$. It characterizes $\Sigma$ since $\Sigma=\left\{z \in A^{\mathbb{N}} \mid \forall w \sqsubset z, w \in \mathcal{L}(\Sigma)\right\}$.

A subshift $\Sigma$ is nilpotent if there is some $k \in \mathbb{N}$ such that $\sigma^{k}(\Sigma)=\left\{0^{\omega}\right\}$. Equivalently, $\Sigma \subset A^{k} 0^{\omega}$ for some $k$. In particular, it is finite.

\section{Trace}

Trace. In this section we define the main notion introduced in the paper, namely the trace of a CA.

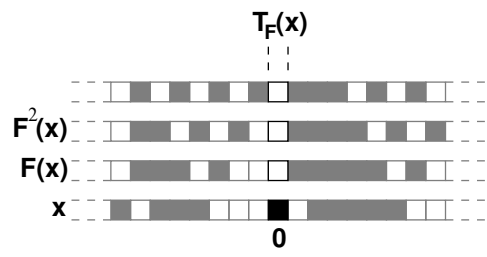

Fig. 1. The trace seen on the space-time diagram.

Definition 1 (Trace). Given a $C A F$, the trace of $F$ with initial condition $x \in A^{\mathbb{Z}}$ is $T_{F}(x)=\left(F^{j}(x)_{0}\right)_{j \in \mathbb{N}}$. In other words, it is the central column of the space-time diagram of initial configuration $x$ (see Figure 1). The trace subshift of $F$ is $\tau(F)=T_{F}\left(A^{\mathbb{Z}}\right)$. Similarly, we define $T_{F}^{k}(x)=\left(F^{j}(x)_{[0, k-1]}\right)_{j \in \mathbb{N}}$ which is the sequence of the words at positions in $[0, k-1]$ in the space-time diagram of initial configuration $x$.

For instance, the trace subshift of the shift is $(0+1)^{\omega}$, it is $0^{\omega}+1^{\omega}$ for the identity and $(0+1) 0^{\omega}$ for the null CA (see [10] for more examples).

Note that $T_{F} F=\sigma T_{F}$ and, as $T_{F}$ is continuous, it is a factorization between the CA and the one-sided full shift which means that their two dynamics are very closely related. For more about factorization, see [11]. 
Subtrace. If $\Sigma$ is a subshift on an alphabet $B \subset A^{k}$, and $i \in[0, k-1]$, then for all $z=\left(\left(z_{j}\right)_{0} \ldots\left(z_{j}\right)_{k-1}\right)_{j \in \mathbb{N}} \in \Sigma$, we define its $i^{\text {th }}$ projection as $\pi_{i}(z)=\left(\left(z_{j}\right)_{i}\right)_{j \in \mathbb{N}} \in$ $A^{\mathbb{N}}$, and $\pi_{[n, m]}(z)=\left(\left(z_{j}\right)_{n}, \ldots,\left(z_{j}\right)_{m}\right)_{j \in \mathbb{N}}$. We also note $\pi(\Sigma)=\bigcup_{0 \leq i<k} \pi_{i}(\Sigma)$, which is a subshift on $A$.

Definition 2. Given a $C A F$ on the alphabet $B \subset A^{k}$, the subtrace subshift is defined by $\stackrel{\circ}{\tau}(F)=\bigcup_{0 \leq i<k}\left\{\left(\left(F^{j}(x)_{0}\right)_{i}\right)_{j \in \mathbb{N}} \mid x \in B^{\mathbb{Z}}\right\}=\pi(\tau(F))$.

\section{The nilpotency problem}

Definition 3. Consider a $C A F$ of local rule $f: A^{d} \rightarrow A$ and $q \in A$. A state $q \in A$ is spreading if for all word $u \in A^{d}$ containing letter $q$, we have $f(u)=q$. $A C A$ on alphabet $A$ is q-nilpotent (or simply nilpotent) if there is some integer $j$ for which $\forall x \in A^{\mathbb{Z}}, F^{j}(x)={ }^{\omega} q^{\omega}$.

Note that a CA is nilpotent if and only if its trace is nilpotent.

Theorem 1 (Kari[5]). The problem

Instance: a $C A F$ with a spreading state $q$

Question: is F q-nilpotent?

is undecidable.

Note that it is still undecidable if we restrict to OCA since two CA with the same diameter and same local rule are either both nilpotent or both non nilpotent whatever are their anchor. If the first CA $F$ has anchor $m$ and the second CA $F^{\prime}$ has anchor $m^{\prime}$ and if there is a $k$ such that for all configuration $x, F^{k}(x)={ }^{\omega} q^{\omega}$ then $F^{\prime k}(x)=\sigma^{k\left(m-m^{\prime}\right)}\left(F^{k}(x)\right)={ }^{\omega} q^{\omega}$.

Proposition 1. An OCA with a spreading state $q$ is q-nilpotent if and only if $q$ appears in all its space-time diagrams.

Proof. Consider a CA F. If its diameter is 1 (each cell uses only its state for the local rule), then the equivalence is trivial since for all letter $a$, the spacetime diagram for ${ }^{\omega} a^{\omega}$ contains a $q$ after $k_{a}$ steps and hence for all configuration $x, F^{\max _{a \in A} k_{a}}(x)={ }^{\omega} q^{\omega}$. Otherwise, for every configuration $x \in A^{\mathbb{N}}$, there are some integers $i$ and $j$ such that $F^{j}(x)_{i}=q$. Note that, by spreadingness, $F^{j+\left\lceil\frac{i}{d}\right\rceil}(x)_{0}=$ $q$. Hence $\bigcup_{j \in \mathbb{N}} F^{-j}([q])$ is a covering by open subsets of the compact $A^{\mathbb{N}}$, so we can find some $k \in \mathbb{N}$ such that $A^{\mathbb{N}}=\bigcup_{j \leq k} F^{-j}([q])=F^{-k}([q])$. By shiftinvariance, $F^{k}\left(A^{\mathbb{N}}\right)=\left\{{ }^{\omega} q^{\omega}\right\}$.

Proposition 2. Let $F$ a $C A$ on alphabet $A$, of diameter d, and with a spreading state $q \in A$. We can build a $C A F^{\prime}$ on alphabet $\{0,1\}^{k}$ for some $k \in \mathbb{N}^{*}$, such that $F^{\prime}$ is $(0, \ldots, 0)$-nilpotent if and only if $F$ is q-nilpotent. 
Proof. Let $k=\left\lceil\log _{2}|A|\right\rceil$ and $\phi: A \rightarrow\{0,1\}^{k}$ an injection such that $\phi(q)=$ $(0, \ldots, 0) . F^{\prime}$ can be defined by the same diameter and anchor as $F$, and local rule:

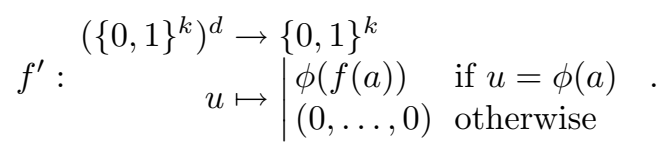

\section{Subtrace problems}

A subtrace is somewhat the trace of a "CA" which does not apply the same evolution rule on each cell. We will first prove, by reduction from the nilpotency problem, the undecidability of a certain class of properties over subtrace subshifts, that is, non-trivial nilpotent-stable properties. In the next section, we will reduce properties over trace subshifts.

\subsection{The full subtrace problem.}

This section is devoted to reducing the nilpotency problem to the problem FULL:

Instance: an OCA on alphabet $A^{k+1}$

Question: is its subtrace equal to the full shift $A^{\mathbb{N}}$ ?

Our method presents similarities with [8].

Let $F$ a OCA on $A$ of local rule $f$, diameter $d$, and with a spreading state $q \in A$. From Proposition 2, we can assume without loss of generality that $A=$ $\{0,1\}^{k}$ for some $k \in \mathbb{N}^{*}$, and $q=(0, \ldots, 0)$. We can build the OCA $\sigma \odot F$ on alphabet $\{0,1\}^{1+k}$ with the local rule:

$$
\begin{aligned}
\left(\{0,1\} \times\{0,1\}^{k}\right)^{d} & \rightarrow\{0,1\} \times\{0,1\}^{k} \\
\left.g:\left(\left(a_{0}, x_{0}\right) \ldots\left(a_{d-1}, x_{d-1}\right)\right)\right) & \mapsto \mid \begin{array}{ll}
\left(a_{1}, f\left(x_{0} \ldots x_{d-1}\right)\right) & \text { if } x_{0} \neq q \\
(0, q) & \text { otherwise }
\end{array}
\end{aligned}
$$

This rule works almost like the Cartesian product of the shift on $\{0,1\}$ and $F$ except that it applies the shift only if the state of the cell for $F$ is not $q$ and else goes to 0 . It behaves as if $F$ is supplying power to the shift $\mathrm{CA}$ and $q$ means "no power".

Lemma 1. If $F$ is q-nilpotent, then $\stackrel{\circ}{\tau}(\sigma \odot F)$ too. Otherwise, $\stackrel{\circ}{\tau}(\sigma \odot F)=A^{\mathbb{N}}$.

Proof. We can see that the last $k$ projections exactly represent the application of the CA $F$. In particular, if $F$ is $q$-nilpotent, then there is some $j \in$ $\mathbb{N}$ for which $\forall x \in A^{\mathbb{Z}}, \pi_{[1, k]}\left(T_{\sigma \odot F}(x)\right)_{[j,+\infty)}=q^{\omega}$, and from the rule we get $\pi_{0}\left(T_{\sigma \odot F(x)}\right)_{[j+1,+\infty)}=0^{\omega}$, so $\stackrel{\circ}{\tau}(\sigma \odot F)$ is $(0, q)$-nilpotent.

On the other hand, if $F$ is not $q$-nilpotent, then from Proposition 1, one can find a configuration $x \in A^{\mathbb{Z}}$ such that $\forall j \in \mathbb{N}, \forall i \in \mathbb{Z}, F^{j}(x)_{i} \neq q$. For every $z \in A^{\mathbb{N}}$, define $y \in\left(\{0,1\}^{1+k}\right)^{\mathbb{Z}}$ such that $\pi_{[1, k]}(y)=x$ and $\pi_{0}(y)_{[0,+\infty)}=z$. The second case of the rule will never be applied in the orbit of $y$; hence $\pi_{0}\left(T_{\sigma \odot F(y)}\right)=z$. 
Theorem 2. Problem Full is undecidable.

Proof. $\sigma \odot F$ is clearly computable from $F$, so if we could decide whether $\stackrel{\circ}{\tau}(\sigma \odot F)$ is $A^{\mathbb{N}}$, then from Lemma 1 , we could decide whether $F$ is nilpotent.

\subsection{Other problems.}

In this section, we use the previously-built $\mathrm{CA}$ in order to reduce the nilpotency problem to a large class of problems over the subtrace of a CA on $\{0,1\}^{2+k}$.

Definition 4. A property $P$ on subshifts is nilpotent-stable if for every subshift $\Sigma$ and every nilpotent subshift $H, \Sigma \in P \Leftrightarrow \Sigma \cup H \in P$.

By non-trivial property, we mean that some OCA traces respect it and some do not.

Theorem 3. For any non-trivial nilpotent-stable property $P$, the problem

Instance: a $C A F$ on alphabet $\{0,1\}^{k}$, with $k \in \mathbb{N}^{*}$

Question: has the subtrace subshift $\stackrel{\circ}{\tau}(F)$ property $P$ ?

is undecidable.

Proof. Let $P$ be a non-trivial nilpotent-stable property of the subshifts of $A^{\mathbb{N}}$. Should we take its complementary, we can assume $P$ is false for the full shift $A^{\mathbb{N}}$. Let $H$ be an OCA on $\{0,1\}$ such that $\tau(H) \in P, h$ its local rule and $d$ its diameter. Let $F$ be an OCA on $A^{k}$ of local rule $f$, diameter $d$ and with spreading state $q$. Without loss of generality, $A=\{0,1\}$ and $q=(0, \ldots, 0)$. We can build the OCA $G=(\sigma \circ F) \times H$ on alphabet $\{0,1\}^{2+k}$ with the local rule:

$$
\begin{aligned}
\left(\{0,1\} \times\{0,1\}^{k} \times\{0,1\}\right)^{d} & \rightarrow\{0,1\} \times\{0,1\}^{k} \times\{0,1\} \\
g:\left(\left(a_{0}, x_{0}, b_{0}\right) \ldots\left(a_{d-1}, x_{d-1}, b_{d-1}\right)\right) & \mapsto \begin{array}{r}
a_{1}, f\left(x_{0} \ldots x_{d-1}\right), h\left(b_{0} \ldots b_{d-1}\right) \\
\text { if } x_{0} \neq q \\
\left(0, q, h\left(b_{0} \ldots b_{d-1}\right)\right) \text { otherwise }
\end{array}
\end{aligned}
$$

The first $1+k$ projections are the previously built automaton $\sigma \odot F$, and the last one represents $H$. Hence, $\stackrel{\circ}{\tau}(G)=\stackrel{\circ}{\tau}(\sigma \odot F) \cup \tau(H)$, and we can use Lemma 1. If $F$ is nilpotent, then $\stackrel{\circ}{\tau}(\sigma \odot F)$ too, and $\stackrel{\circ}{\tau}(G)$ respects $P$ since $\tau(H)$ does, and $P$ is nilpotent-stable. Otherwise, $\stackrel{\circ}{\tau}(\sigma \odot F)=A^{\mathbb{N}}$ does not respect $P$. Hence, $\stackrel{\circ}{\tau}(G)$ respects $P$ if and only if $F$ is $q$-nilpotent. $G$ is clearly computable from $F$, so if we could decide $P$, then we could decide whether $F$ is nilpotent.

\section{From subtrace to trace}

In this section, we assume $G$ is an OCA on $A^{k}$, with $A=\{0,1\}$, and we want to build a $\mathrm{CA}$ on $A$ that simulates it in a certain way, thus transforming subtrace into trace, provided a little restriction. This construction is similar to that done 
in [10]. Remark that it is well known that $G$ can be simulated by a CA $F$ on $A$, as soon as its diameter is wide enough. Each cell can see its neighborhood as words of $A^{k}$ and evolve accordingly. The problem is that all cells must have the same local rule, so they have to find from the neighborhood which "column" of the $A^{k}$ simulation they are representing. This can be achieved by delimiting meaningful words with a border word $10^{k}$. That simulation allows to "see" the evolution of $G$ as an evolution of $F$. We want a little more: all evolutions of $F$ must not be far from an evolution of $F$, especially those that do not correspond to alternating meaningful words and borders words.

We have two execution modes: a simulation mode will simulate properly the execution of the OCA $G$ on alphabet $A^{k}$, and a default mode will be applied if the neighborhood contains invalid information. All modes must have an evolution that locally "looks like" some evolution of $G$, and in particular we have to ensure that when a mode is applied to a cell, the same mode keeps being applied there in the following generations, because a change of mode would produce an invalid trace.

Macrocells. A concatenation of a word of $A^{k}$ and of the border word $10^{k}$ will be called a macrocell. Let $B=A^{k} 10^{k} \subset A^{h}$ the set of macrocells, where $h=2 k+1$. By construction, it has the interesting property that it cannot overlap itself on more that half of its length.

Property 1. If $0<i \leq k$, then the intersection $B A^{i} \cap A^{i} B$ is empty (equivalently $\left.[B] \cap[B]_{i}=\emptyset\right)$.

Simulation mode. We will assume in the rest of the section that $G$ has a diameter 2 (this can be easily generalized), and local rule $g$.

The simulation mode will be applied to macrocells that are not overlapped by another macrocell on their right. For this purpose, they have to "look" on their right (in the local rule). Let $\Theta=B A^{h} \backslash \bigcup_{0<i<h} A^{i} B A^{h-i}$ the set of macrocells followed by something that in no way could be understood as an overlapping macrocell. The first thing to notice is that this set is not empty, since it contains successions of two macrocells thanks to Property 1.

Lemma 2. $B B \subset \Theta$.

Proof. If $0<i \leq k$, then $B B \cap A^{i} B A^{h-i} \subset\left(B A^{i} \cap A^{i} B\right) A^{h-i}=\emptyset$. If $k<i<h$, then $B B \cap A^{i} B A^{h-i} \subset A^{i}\left(A^{h-i} B \cap B A^{h-i}\right)=\emptyset$.

We can now define a rule on the set $\Theta$ that represents the evolution of such a macrocell that sees on its right either a macrocell abut, or at least no overlapping macrocell:

$$
\begin{aligned}
\Theta A^{h} & \rightarrow B \\
\Delta: \quad u & \mapsto \mid \begin{array}{ll}
g\left(u_{[0, k-1]}, u_{[h, h+k-1]}\right) 10^{k} & \text { if } u \in B \Theta \\
g\left(u_{[0, k-1]}, u_{[0, k-1]}\right) 10^{m} & \text { otherwise }
\end{array} .
\end{aligned}
$$


Default mode. In the case where the neighborhood is not understandable as a valid word, we have to apply a default rule: the null CA, which has the interesting property that it erases the border words that could potentially be in an invalid place.

Combining simulation mode and default mode, we are now able to turn the function $\Delta$ into a local rule on $\{0,1\}$. Indeed, we can define, for anchor $m=h-1$ and diameter $d=4 h-1$ :

$$
\begin{aligned}
& \{0,1\}^{d} \rightarrow\{0,1\} \\
& f: \quad w \mapsto \mid \begin{array}{ll}
\Delta(u)_{i} & \text { if } w \in A^{m-i} u A^{i}, \text { for some } u \in \Theta A^{h}, i \in[0, h-1], \\
0 & \text { otherwise }
\end{array}
\end{aligned}
$$

since such an integer $i$ and such a word $u$ would be unique (from the construction of $\Theta$ ). This local rule is such that $f\left(A^{m} u A^{m}\right)=\Delta(u)$ for every $u \in \Theta A^{h}$, which is what we wanted: it can simulate in one step the behavior of our CA $G$. Let $F$ the corresponding rule. You can also note that $F([\Theta])=\left[\Delta\left(\Theta A^{h}\right)\right] \subset[B]$ by definition, and that $F^{-1}([1]) \subset \bigcup_{0 \leq j \leq m}[\Theta]_{-j}$ since the default mode only produces zeros.

Stability. The following lemmas guarantee that no cell changes its evolution mode.

Lemma 3. $F^{-1}([B])=[\Theta]$.

Proof. By construction, $F^{-1}([B]) \subset F^{-1}\left([1]_{m}\right) \subset \bigcup_{0 \leq j \leq m}[\Theta]_{-j}$. Now let $x \in$ $F^{-1}([B])$. Then, for some $j \in[0, m], F(x) \in[B] \cap F\left([\Theta]_{-j}\right) \subset[B] \cap[B]_{-j}$. By Property 1 , we conclude that $j=0$, i.e. $F^{-1}([B]) \subset[\Theta]$. Conversely, we have already seen that $[\Theta] \subset F^{-1}([B])$.

Lemma 4. $F([\Theta]) \subset[\Theta]$.

Proof. By definition of $[\Theta]$, it is included in $\bigcap_{0<i<h}[\Theta]_{i}^{C}$. Moreover, $F\left([\Theta]^{C}\right) \subset$ $[B]^{C}$ by Lemma 3. Combining the two, we get $F([\Theta]) \subset \bigcap_{0<i<h}[B]_{i}^{C}$. Hence, $F([\Theta]) \subset F([B]) \backslash \bigcup_{0<i<h}[B]_{i}=[\Theta]$.

Trace. The fact the evolution modes are stable helps us conclude the simulation result.

Lemma 5. If $y \in\left(A^{m}\right)^{\mathbb{N}}$ and $x \in A^{\mathbb{N}}$ such that $\forall i \in \mathbb{N}, x_{[i h, i h+m-1]}=y_{i} \in A^{m}$ and $x_{[i h+m,(i+1) h-1]}=10^{m}$, then $T_{F}^{m}(x)=T_{G}(y)$.

Proof. We can prove by induction on the generation $j \in \mathbb{N}$, that for any $i \in \mathbb{N}$, $F^{j}(x)_{[i h,(i+1) h-1]}=G^{j}(y)_{i} 10^{m}$. This property holds for $j=0$. Now suppose it is true at generation $j \in \mathbb{N}$, and let us prove it for generation $j+1$. Let $i \in \mathbb{N}$. $F^{j}(x) \in[B B]_{i h} \subset \Theta$ from the induction hypothesis and Lemma 2. Therefore, we are in execution mode between cells $i h$ and $(i+1) h$ :

$$
\begin{aligned}
F^{j+1}(x)_{[i h,(i+1) h-1]} & =\Delta\left(F^{j}(x)_{[i h,(i+1) h-1]}, F^{j}(x)_{[(i+1) h,(i+2) h-1]}\right) \\
& =\Delta\left(F^{j}(y)_{i}, F^{j}(y)_{i+1}\right)=F^{j+1}(y)_{i}
\end{aligned}
$$

In particular, $T_{F}^{m}(x)=\left(F^{j}(y)_{[0, m-1]}\right)_{j \in \mathbb{N}}=T_{G}(y)$. 
Lemma 6. If $p \in \mathbb{N}$ and $x \in A^{\mathbb{N}}$ are such that $\forall i<p, x_{[i h,(i+2) h-1]} \in \Theta$ and $x_{[p h,(p+2) h-1]} \notin \Theta$; let $y \in\left(A^{m}\right)^{\mathbb{N}}$ the ultimately uniform infinite word such that $\forall i<p, y_{i}=x_{[i h, i h+m-1]}$ and $\forall i \geq p, y_{i}=x_{[(p-1) h,(p-1) h+m-1]}$. Then $T_{F}^{m}(x)=$ $T_{G}(y)$.

Proof. Similarly to the proof of Lemma 5 , it can easily be proved by induction on the generation $j \in \mathbb{N}$ that for any $i<p, F^{j}(x)_{[i h,(i+1) h-1]}=G^{j}(y)_{i} 10^{m}$, since execution is always applied. Hence, $T_{F}^{m}(x)=T_{G}(y)$.

Lemma 7. The trace of $F$ is $\stackrel{\circ}{\tau}(G) \cup \Sigma$, where $\Sigma \subset\left\{0^{\omega}, 10^{\omega}, 1^{\omega}\right\}$.

Proof. Let $x \in A^{\mathbb{Z}}$.

- If $x \in[\Theta]_{-q}$ for some $q \in[0, m-1]$, and $\forall i \in \mathbb{N}, x_{[i h,(i+2) h-1]} \in \Theta$, and, from Lemma $5, T_{F}(x)=\pi_{i}\left(T_{G}(y)\right)$, where $y_{i}=x_{[i h, i h+m-1]}$.

- If $x \in[\Theta]_{-q}$ for some $q \in[0, m-1]$, and there is some integer $p$ such that $\forall i<p, x_{[i h,(i+2) h-1]} \in \Theta$ and $x_{[p h,(p+2) h-1]} \notin \Theta$, then from Lemma 6 , $T_{F}(x)=\pi_{i}\left(T_{G}(y)\right)$, for some $y \in\left(A^{m}\right)^{\mathbb{N}}$.

- If $x \in[\Theta]_{-q}$ for some $q \in[m, h-1]$, then by induction and Lemma 4, we can see that $\forall j \in \mathbb{N}, F^{j}(x) \in[\Theta]_{-q}$. Thus, $\forall j \in \mathbb{N}, F^{j}(x)_{0}=1$ if $q=m$ and 0 if $m<q<h$.

- Lastly, if $x \notin[\Theta]_{-q}$ for any $q \in[0, h-1]$, then default mode is applied, and, according to Lemma 3 , will always be: $\forall j>0, F^{j}(x)_{0}=0$.

Putting things together, the first two cases give $\pi(\tau(G))$ and the last two give $\left\{0^{\omega}, 10^{\omega}, 1^{\omega}\right\}$.

We are thereby able to "simulate" a OCA $G$ on alphabet $A^{m}$ by a CA $F$ on alphabet $A$. This simulation transforms the subtrace of $G$ into the trace of $F$, provided a little restriction - adding three obviously nilpotent infinite words.

Theorem 4. For any non-trivial nilpotent-stable property $P$, the problem

Instance: $a C A$ on alphabet $\{0,1\}$

Question: has its trace subshift property $P$

is undecidable.

Proof. Let $P$ a non-trivial nilpotent-stable property. From any OCA $G$ on alphabet $\{0,1\}^{m}$, we can, using previous construction, computably build a CA $F$ on alphabet $\{0,1\}$ such that $\tau(F)=\stackrel{\circ}{\tau}(G) \cup \Sigma$, where $\Sigma=\left\{0^{\omega}, 10^{\omega}, 1^{\omega}\right\}$ are nilpotent subshifts. In particular, we can notice that $\stackrel{\circ}{\tau}(G)$ respects $P$ if and only if $\tau(F)$ does. So if we could decide, from the entry $F$, whether $\tau(F)$ respects $P$, then we could decide from the entry $G$ whether $\stackrel{\circ}{\tau}(G)$ does. 


\section{Conclusion}

We have proved the undecidability of a class of problems over CA traces, which could lead us towards a Rice-like Theorem. This class is very comprehensive: fullness, finiteness, ultimate periodicity, soficness, finite type, inclusion of a particular word as a factor... are all nilpotent-stable non-trivial properties. On the other hand, note that context-sensitivity of the language is trivial since true for all trace subshifts according to [12].

The perspectives now would be to decrease the amount of problems that are not concerned. Finite properties (which depend only on the $k^{\text {th }}$ prefix of the subshift, for some $k \in \mathbb{N}$ ) are clearly decidable. Are they the only ones?

Our result can be easily adpated to traces of any fixed width of a CA on any given alphabet, but with arbitrarily wide neighborhoods. Another open problem would be to widen our result to study properties of the canonical factor (of width $d)$, or at least with fixed diameter (and arbitrary alphabet). That would, in particular, comprehend the undecidability of the language classification, proved

in [13]. Nonetheless, our construction can hardly be generalized for that matter, since it is based on increasing the diameter to put more information.

\section{References}

1. Gilman, R.H.: Classes of linear automata. Erg. Th. \& Dyn. Sys. 7 (1988) 105-118

2. Hurley, M.: Attractors in cellular automata. Erg. Th. \& Dyn. Sys. 10 (1990) $131-140$

3. Mazoyer, J., Rapaport, I.: Inducing an order on cellular automata by a grouping operation. In: STACS. Volume 1373 of LNCS. (1998) 116-127

4. Kůrka, P.: Languages, equicontinuity and attractors in cellular automata. Erg. Th. \& Dyn. Sys. 17 (1997) 417-433

5. Kari, J.: The nilpotency problem of one-dimensional cellular automata. SIAM J. on Computing 21(3) (1992) 571-586

6. Kari, J.: Rice's theorem for the limit sets of cellular automata. Th. Comp. Sci. 127(2) (1994) 229-254

7. Durand, B., Formenti, E., Varouchas, G.: On undecidability of equicontinuity classification for cellular automata. In Morvan, M., Rémila, E., eds.: DMCS'03. Volume AB of DMTCS Proc., Disc. Math. and Th. Comp. Sci. (2003) 117-128

8. Ollinger, N.: The intrinsic universality problem of one-dimensional cellular automata. In: STACS. Volume 2607 of LNCS., Springer-Verlag (2003) 632-641

9. Cervelle, J., Durand, B.: Tilings: recursivity and regularity. Th. Comp. Sci. 310(13) (2004) 469-477

10. Cervelle, J., Formenti, E., Guillon, P.: Sofic trace of a cellular automaton. In: Computability in Europe. Lecture Notes in Computer Science, Siena, Italy, SpringerVerlag (jun 2007)

11. Kůrka, P.: Topological and symbolic dynamics. Société Mathématique de France (2003)

12. Gilman, R.H.: Notes on cellular automata. manuscript (1988)

13. di Lena, P.: Decidable and Computational properties of Cellular Automata. PhD thesis, Università di Bologna e Padova (dec 2006) 